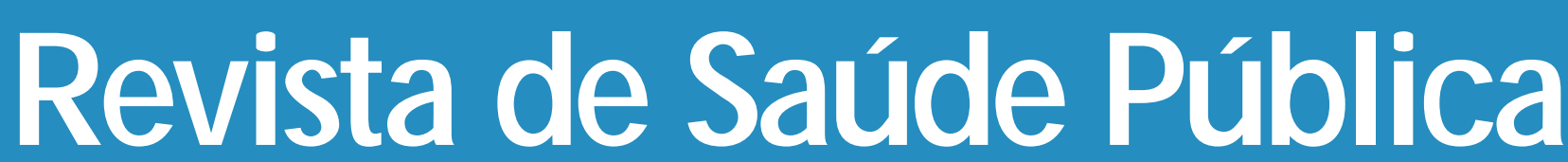

$\begin{array}{llllllll}\mathbf{J} & \mathbf{O} & \mathbf{U} & \mathbf{R} & \mathbf{N} & \mathbf{A} & \mathbf{L}\end{array}$

$0 F$

$\mathbf{P} \mathbf{U}$

B L I C

H E A L T

\title{
Prevalencia y factores de riesgo asociados a sífilis en mujeres
}

\section{Prevalence and risk factors associated with syphilis in women}

Carlos A. Hernández-Girón, Aurelio Cruz-Valdez, Luis Juárez Figueroa y Mauricio Hernández-Avila

Centro de Investigaciones en Salud Poblacional do Instituto Nacional de Salud Pública (INSP). Cuernavaca, Mor. M éxico (C.A. H.G., A. C.V., M.H.A.), Centro de Investigaciones

sobre Enfermedades Infecciosas do INSP. Cuernavaca, Mor. México (L.J.F.)

HERN AN DEZ-G IRO N Carlos A., Aurelio Cruz-Valdez, Luis Juárez Figueroa Prevalencia y factores de riesgo sociados a sífilis en mujeres Rev. Saúde Pública, 32 (6): 579-86, 1998

(c) Copyright Faculdade de Saúde Pública da USP. Proibida a reprodução mesmo que parcial sem a devida autorização do Editor Científico. Proibida a utilização de matérias para fins comerciais. All rights reserved. 


\title{
Prevalencia y factores de riesgo asociados a sífilis en mujeres
}

\section{Prevalence and risk factors associated with syphilis in women}

\author{
Carlos A. Hernández-G irón, Aurelio Cruz-Valdez, Luis Juárez Figueroa \\ y Mauricio Hernández-Avila \\ Centro de Investigaciones en Salud Poblacional do Instituto Nacional de Salud Pública (INSP). \\ Cuernavaca, Mor. México (C.A. H.G., A. C.V., M.H.A.), Centro de Investigaciones \\ sobre Enfermedades Infecciosas do INSP. Cuernavaca, Mor. México (L.J.F.)
}

\section{Resumen}

Introducción

Se ha demostrado que la sífilis, debido a la ulceración genital que produce, es un cofactor asociado para adquirir otras enfermedades de transmisión sexual (ETS), principalmente de origen viral como herpes tipo-2, hepatitis B, y el VIH. Aunque las mujeres trabajadoras del sexo comercial (MTSC) han adquirido mejores conocimientos para prevenir las ETS, constituyen un grupo que por su heterogenicidad en términos de condición socioeconómica, estado de salud, ambiente y sitio de trabajo, manifiestan diferentes actitudes y conocimientos que hacen latente la posibilidad de adquirir y transmitir ETS incluyendo sífilis, por lo que lo estudio hace un acercamiento hacia los factores asociados a infección por Treponema pallidum en este grupo de mujeres.
\end{abstract}

Método Basado en un marco muestral, que identifica sitios donde se practica el comercio sexual femenino en la ciudad de México, se seleccionó una muestra de 807 MTSC, a quienes previo consentimiento informado, se entrevistó para que respondieran un cuestionario estructurado. Se obtuvo una muestra sanguínea para la identificación de diversos marcadores serológicos de ETS de acuerdo al manual de procedimientos para el diagnóstico de ETS. Para el diagnóstico de Treponema pallidum se utilizó una prueba de tamizaje de RPR (Bigaux Diagnóstica), y prueba confirmatoria de FTA-ABS (Pasteur Diagnostics).

Resultados

Las prevalencias de sífilis en la muestra de MTSC fue de 6,4\% (52/807), siendo mayor en quienes trabajaban en sitios de calle comparadas con aquellas de estéticas. La edad de las mujeres entrevistadas osciló entre 17 y 58 años con una media de 29,2 años (d.s. 7,3 años). La prevalencia de sífilis fue mayor en los grupos etáreos mayores de 30 anos. La edad de inicio de relaciones sexuales varió desde 11 hasta 30 anos con una media de 16 años (d.s. 3,1 años). Los factores predictores de infección por T. pallidum, determinados mediante regresión logística ajustada, fueron: sitio de trabajo (bar y puntos de calle), NSE (medio y bajo), edad (mayores de 30 anos), antiguedad en el trabajo sexual (> 5 años), y número de clientes en una semana $(>10)$.

Correspondencia para/Correspondence to : Carlos A. Hernández-Girón - Av. Universidad \# 655 Col. Sta. María Ahuacatitlán. C.P. 62508 Cuernavaca, Mor. México. E-mail: chernand@insp3.insp.mx Recibido en 18.11.1997. Aprobado en 5.6.1998. 
Conclusiones

Introduction

Method

Results

Conclusion
A pesar de las limitaciones de precisión estadística, queda demostrado que existe una heterogenicidad de MTSC, diferenciado principalmente por el sitio donde se desempeñan. Debe entenderse que más que grupos de riesgo de adquirir y transmitir ETS, existen prácticas sexuales de riesgo en cualquier individuo que tiene relaciones sexuales, que aunadas a infecciones predisponentes como sífilis, facilitan la transmisibilidad de otras ETS. Por lo tanto, las campañas de prevención y fomento de uso de condón, deben orientarse no sólo a las MTSC sino también a sus clientes y parejas, con la finalidad de que todos asuman la responsabilidad del sexo seguro.

Sífilis, prevención. Prostitución. Infecciones oportunistas. Conducta sexual.

\section{Abstract}

Although the incidence of syphilis is generally low, it remains an important global public health problem, given its interaction with other sexually transmitted diseases (STDs). It has been shown that syphilis, due to the genital ulcers it produces, is a co-factor for acquiring other STDs, principally those of viral origin such as herpes simples type 2, hepatitis B and HIV. Many female commercial sex workers (FCSW) in Mexico have been found to have acquired good levels of knowledge about STD prevention. Nevertheless, they constitute a heterogeneous group in terms of socio-economic level, health status and type of work site; these factors in turn appear to determine their attitudes, knowledge and behavior related to acquiring and transmitting STDs, including syphilis. This study, therefore, focused on the factors associated with Treponema pallidum infection in this group of women.

Based on a sample frame of sites where female commercial sex work takes place within Mexico City, a sample of 807 FCSWs was selected; after providing informed consent, they completed a structured questionnaire. A blood sample for identifying serologic markers for STDs was collected and analyzed according to a procedure manual for STD diagnosis. Treponema pallidum was diagnosed using the RPR (Bigaux Diagnostica) screening test, and FTA - ABS (Pasteur Diagnostics) for confirmation.

The prevalence of syphilis in this sample of FCSWs was 6.4\% (52/807), and was higher among women who worked at street sites than among those who worked in massage parlors. The age of the women interviewed ranged from 17 to 58 years, with a mean of 29.2 years (SD 7.3 years); syphilis was more prevalent among women over 30 years of age. Age at first sexual intercourse ranged from 11 to 30 years, with a mean of 16 years (SD 3.1 years), which is similar to that of the general female population in Mexico. Predictive factors for $T$. pallidum infection, determined adjusted logistic regression, included: tupe of by work site (bar and street sites); socio-economic level (middle and low); age (over 30 years); duration of involvement in sex work (> 5 years) and number of clients per week (>10).

In spite of some limitations regarding statistical precision, this study shows that FCSWs are heterogeneous in terms of risk of acquiring STDs, including syphilis; the principal differentiating factor was shown to be the type of work site. Given that it is not belonging to a risk group but rather participating in risky practices that leads to acquiring STDs, situations that facilitate riskier or safer practices (such as type of sex work site, for sex workers) should be taken into account when studying people's risk level. STD prevention campaigns must also consider these factors, in addition to focusing on FCSWs and their clients and personal partners, so that all involved assume their responsibility for safer sex.

Syphilis, prevention. Prostitution. Opportunistic infections. Sex behavior. 


\section{INTRODUCCIÓN}

Posterior a la Segunda Guerra Mundial, la incidencia de sífilis disminuyó debido a la utilización de la penicilina. Aunque la incidencia de sífilis es baja, continua siendo un importante problema de salud pública a nivel mundial por su interacción con otras enfermedades de transmisión sexual (ETS). Hoy se sabe que la historia natural de la enfermedad puede durar décadas y el diagnóstico del agente etiológico, Treponema pallidum, es definido serológicamente además del diagnóstico clínico y bacteriológico (Zenker \& Rolfs ${ }^{22}$, 1990). Con la aparición de la infección por el virus de la inmunodeficiencia humana (VIH) en el mundo, la efectividad de los régimenes conocidos de tratamiento han sido cuestionados; además, recientemente se ha demostrado que la sífilis, debido a la ulceración genital que produce, es un cofactor asociado para adquirir otras ETS, principalmente de origen viral como herpes tipo 2, hepatitis B, y el VIH (OPS ${ }^{15}$, 1994; Piot $^{17}$ 1990).

Aunque las mujeres trabajadoras del sexo comercial (MTS) han adquirido mejores conocimientos para prevenir las ETS, constituyen un grupo que por su heterogenicidad en términos de condición socioeconómica, estado de salud, ambiente y sitio de trabajo, manifiestan diferentes actitudes y conocimientos que hacen latente la posibilidad de adquirir y transmitir alguna ETS (Estébanez y col. ${ }^{7}, 1993$ ). Por otra parte, la presencia de sífilis en mujeres embarazadas puede ocasionar pérdidas fetales y daños a la salud del recién nacido, con las consecuentes repercusiones psicológicas y sociales $\left(\right.$ Brown $^{4}, 1985$; Rawstron y col. $\left.{ }^{18}, 1993\right)$.

Lo presente trabajo investiga características sociodemográficas, de comportamiento sexual y de conocimientos sobre ETS asociados a infección por sífilis, como una contribución para definir la epidemiología de esta enfermedad en MTSC que permitan reorientar los programas de prevención y control en esta población.

\section{MÉTO DO}

En 1993, basado en un marco muestral que identifica sitios donde se practica el comercio sexual femenino en la ciudad de México, se seleccionó una muestra de 807 MTSC. Se consideraron 3 categorías de sitios: "calle" que incluye mujeres que se encuentran en la vía pública, negociando directamente con los clientes; "bar" que incluye a mujeres que trabajan como meseras en bares, restaurantes y centros nocturnos; y "estética" que incluye mujeres que trabajan en casas particulares, donde se ofrecen servicios de masaje-sauna.

Previo consentimiento informado, las mujeres seleccionadas contestaron un cuestionario estructurado en 4 secciones principales: características sociodemográficas, estado de salud actual y antecedentes de ETS, características de comportamiento sexual y conocimientos sobre SIDA/ETS.

Posteriormente, se obtuvo una muestra sanguínea para la identificación del T. T. palidum de acuerdo al manual de procedimientos para el diagnóstico de ETS (Juárez y col. ${ }^{10}$,1992). Se utilizó una prueba de tamizaje de RPR (Bigaux Diagnóstica), y una prueba confirmatoria de FTAABS (Pasteur Diagnostics). Las pruebas serológicas se llevaron a cabo en los laboratorios del Centro de Investigaciones sobre Enfermedades Infecciosas (CISEI).

Los resultados de los cuestionarios y de las pruebas de laboratorio, se capturaron en una base de datos elaborado en el paquete Fox-Pro versión 2.6 y analizados en los paquetes estadísticos SPSS/pc+ versión 4.0 y EGRET versión 3.0. Se obtuvieron frecuencias simples y medidas de asociación (razones de momios) crudas y ajustadas, valorando su significancia estadística a través de sus intervalos de confianza al $95 \%$.

\section{RESU LTAD OS}

Las prevalencias de sífilis en la muestra de MTSC fue de $6.4 \%$ (52/807) siendo mayor en quienes trabajaban en sitios de calle comparadas con aquellas de estéticas.

La edad de las mujeres entrevistadas osciló entre 17 y 58 años con una media de 29.2 años (d.s. 7,3 años). La prevalencia de sífilis fue mayor en los

Tabla 1 - Comparación de medias según resultado de serología para sífilis en trabajadoras sexuales de la ciudad de M éxico, 1993.

\begin{tabular}{|c|c|c|c|c|c|}
\hline \multirow{2}{*}{$\frac{\text { Característica }}{\text { Edad (años) }}$} & \multicolumn{2}{|c|}{$\begin{array}{c}\text { Sífilis + } \\
\text { media (d.s.) }\end{array}$} & \multicolumn{2}{|c|}{$\begin{array}{c}\text { Sífilis - } \\
\text { media (d.s.) }\end{array}$} & \multirow{2}{*}{$\begin{array}{c}\text { Valor } \\
\text { prueba } p \\
0,048\end{array}$} \\
\hline & 30,2 & $(7,9)$ & 28,2 & $(6,8)$ & \\
\hline Edad Ira. relación sexual (años) & 16,1 & $(3,6)$ & 16,6 & $(2,7)$ & 0,286 \\
\hline $\mathrm{N}$ úmero hijos & 2,7 & $(2,2)$ & 2,3 & $(1,9)$ & 0,117 \\
\hline Antigüedad en el trabajo (años) & 6,3 & $(6,6)$ & 4,7 & $(4,5)$ & 0,049 \\
\hline Días trabajados última semana & 3,9 & $(1,5)$ & 4,0 & $(1,6)$ & 0,707 \\
\hline Clientes en la última semana & 6,7 & $(4,6)$ & 7,4 & $(7,8)$ & 0,631 \\
\hline
\end{tabular}


grupos etáreos mayores de 30 años (Tabla 1). Consecuentemente, se mostró una tendencia estadísticamente significativa de mayor riesgo de seropositividad a sífilis en los grupos etáreos mayores (Tabla 2).

El 59,0\% de las entrevistadas (465/788) eran solteras, $12,0 \%$ (95/788) vivían en unión libre, y 8,8\% (69/788) eran casadas, el 20,2\% restante (159/788) agrupaba a divorciadas, separadas y viudas. Las mujeres casadas mostraron menos riesgo de seropositividad a sífilis, comparado con las solteras (Tabla 2).

Con base a la metodología de Bronfman y col. ${ }^{3}$, 1988 , se creó un indice de nivel socieconómico (NSE), correspondiendo un 45,4\% de las entrevistadas (351/773) a NSE bajo, 31,6\% (244/773) a NSE medio y $23,0 \%(178 / 773)$ a NSE alto. La prevalencia de sífilis fue menor en quienes reflejaron un NSE medio y alto. Las mujeres que correspondieron a un NSE bajo mostraron un riesgo de infección para sífilis de casi el doble, en comparación a aquellas de NSE alto (Tabla 2).
Referente al sitio de trabajo donde se desempeñan, 45,1\% (364/807) lo hacía en puntos de calle, 45,4\% (366/807) en bares y 9,5\% (77/807) en estéticas. La prevalencia de sífilis fue mayor en quienes trabajaban en puntos de calle. Las mujeres que trabajan en puntos de calle mostraron hasta 8 veces más riesgo de infección comparado con las que trabajan en estéticas (Tabla 2).

Por otra parte, la edad de inicio de relaciones sexuales varió desde 11 hasta 30 años con una media de 16 años (d.s. 3,1 años); el riesgo de padecer sífilis disminuye en los grupos que iniciaron relaciones sexuales después de los 15 años. La antigüedad en el trabajo varió (desde uno hasta 30 años con una media de 5,5 años, siendo mayor en el grupo de seropositivas (media 6,3 años), comparado con el grupo de seronegativas (media 4,7 años). Respecto al número de clientes en la última semana previo a la entrevista, no hubo diferencias significativas: media 8 clientes (d.s. 7 clientes) (Tabla 1).

La probabilidad de adquirir sífilis mostró una tendencia significativa de mayor riesgo, conforme

Tabla 2 - Características sociodemográficas asociadas a infección por sífilis en trabajadoras sexuales de la Ciudad de M éxico, 1993.

\begin{tabular}{|c|c|c|c|c|}
\hline Característica & $\mathrm{N} \cong *$ & Prevalencia** & RM*** IC 95 & $p^{* * * *}$ \\
\hline \multicolumn{5}{|l|}{ Edad } \\
\hline 17-23 años & 230 & 4,8 & 1,0 & \\
\hline $24-30 “$ & 302 & 5,91 & $2,06-5,9$ & \\
\hline 31 y más & 262 & 9,02 & $0,08-9,4$ & 0,061 \\
\hline Total & 794 & 7,2 & & \\
\hline \multicolumn{5}{|l|}{ Estado civil } \\
\hline Casada & 69 & 2,9 & 1,0 & \\
\hline Soltera & 465 & 6,72 & $0,45-14,8$ & \\
\hline O tra & 254 & 7,82 & $0,06-16,5$ & 0,309 \\
\hline Total & 788 & 6,3 & & \\
\hline \multicolumn{5}{|l|}{ Escolaridad } \\
\hline Preparatoria/profes. & 163 & 3,0 & 1,0 & \\
\hline Primaria com/ secund. & 391 & 6,6 & 2,20 & $0,9-5,8$ \\
\hline Primaria incom. / analfab. & 242 & 8,63 & $0,11-7,8$ & 0,028 \\
\hline Total & 796 & 6,1 & & \\
\hline \multicolumn{5}{|l|}{ Indice nivel socieconómico } \\
\hline N SE alto & 178 & 3,9 & 1,0 & \\
\hline NSE medio & 244 & 5,3 & $1,6-3,4$ & \\
\hline NSE bajo & 351 & 9,1 & $2,1-9,6$ & 0,016 \\
\hline Total & 773 & 6,1 & & \\
\hline \multicolumn{5}{|l|}{ Sitio de trabajo } \\
\hline Estética & 77 & 1,21 & 1,0 & \\
\hline Bar & 366 & 4,4 & $3,06-71,3$ & \\
\hline Calle & 364 & 9,6 & $8,4-160,1$ & 0,001 \\
\hline Total & 807 & 5,1 & & \\
\hline
\end{tabular}


Tabla 3 - Características de comportamiento sexual asociadas a infección por sífilis en trabajadoras sexuales de la ciudad de M éxico, 1993.

\begin{tabular}{|c|c|c|c|c|c|}
\hline Característica & No* & Prevalencia** & RM*** & IC 95\% & $p^{* * * *}$ \\
\hline \multicolumn{6}{|c|}{ Inicio relaciones sexuales (edad) } \\
\hline 20 y más & 43 & 11,6 & 1,0 & & \\
\hline $15-19 “$ & 283 & 5,3 & 0,4 & $0,2-1,2$ & \\
\hline 11 - 14 años & 90 & 18,4 & 1,8 & $0,6-5,0$ & 0,107 \\
\hline Total & 416 & 11,8 & & & \\
\hline \multicolumn{6}{|c|}{ Antigüedad del trabajo } \\
\hline menos de 1 año & 223 & 4,0 & 1,0 & & \\
\hline 1-5 años & 402 & 5,4 & 1,4 & $0,6-3,2$ & \\
\hline 6 y más “ & 153 & 13,7 & 3,8 & $1,6-9,2$ & 0,001 \\
\hline Total & 778 & 7,7 & & & \\
\hline \multicolumn{6}{|c|}{ Clientes última semana } \\
\hline Ninguno & 350 & 4,6 & 1,0 & & \\
\hline 1 - 10 clientes & 364 & 7,6 & 1,7 & $0,8-3,4$ & \\
\hline 11 y más “ & 93 & 8,6 & 1,9 & $0,7-5,1$ & 0,067 \\
\hline Total & 807 & 6,9 & & & \\
\hline \multicolumn{6}{|c|}{ Clientes regulares en su vida } \\
\hline Ninguno & 335 & 4,8 & 1,0 & & \\
\hline 1 - 10 clientes & 262 & 8,0 & 1,7 & $0,8-3,6$ & \\
\hline 11 y más “ & 210 & 7,1 & 1,5 & $0,7-3,4$ & 0,210 \\
\hline Total & 807 & 6,6 & & & \\
\hline \multicolumn{6}{|c|}{ U so condón últimos 3 clientes } \\
\hline Consistente & 432 & 8,6 & 1,0 & & \\
\hline Inconsistente & 145 & 4,1 & 0,4 & $0,2-1,2$ & \\
\hline Sin dato & 221 & 3,6 & 0,4 & $0,2-0,9$ & \\
\hline No usó & 9 & 11,1 & 1.3 & $0,1-11,0$ & 0,018 \\
\hline Total & 807 & 6,8 & & & \\
\hline
\end{tabular}

* Los totales varian debido a los valores perdidos (missing).

** Prevalencia de Sífilis (FTA-ABS)

*** Razón de Momios Cruda

**** Prueba de Tendencias

IC- Intervalo de confianza

se incrementaban los años de ejercer el trabajo, de hasta 4 veces en quienes tenían una antigüedad de 6 años y más. A mayor número de clientes también mayor riesgo de infección, aunque las estadísticas no fueron significativas (Tabla 3 ).

Referente a los servicios sexuales solicitados por el último cliente, el $84 \%$ (487/576) refirió al coito vaginal. De las MTSC entrevistadas, el 20,8\% (153/ 736) refirió haber padecido alguna ETS en toda su vida y 6,0\% (44/739) refirió alguna ETS en el último año.

Finalmente, mediante regresión logística se determinaron que los principales predictores al riesgo para infección por T. pallidum en este grupo de mujeres fueron: edad (mayores a 25 años), NSE (medio y bajo), sitio de trabajo (bar, puntos de calle), antigüedad en el trabajo (mayor a 5 años) y clientes en la última semana (más de 10) (Tabla 4).

\section{DISCUSIÓN}

Con los inicios de la epidemia del SIDA a principios de los 80's, los homosexuales y las MTSC se constituyeron como grupos de alto riesgo para la transmisión de ETS, llevándolos incluso a la estigmatización con leyes restrictivas que incluían exámenes y pruebas de laboratorio obligatorias. Diversos estudios han concluido que la transmisibilidad de ETS en las MTSC se debe más que al número de clientes, a ciertas prácticas riesgosas como drogadicción intravenosa, compañeros sexuales infectados con alguna ETS, sexo anal y relaciones sexuales sin condón (Gómez y col. ${ }^{9}$, 1990; Catania y col. ${ }^{5}, 1992$; Andrus y col. ${ }^{2}, 1990$ ).

Otros estudios realizados en MTSC, han mostrado diversas prevalencias de sífilis y otras ETS, que hacen difícil establecer comparaciones entre grupos. 
Tabla 4 - Características sociodemográficas y de comportamiento sexual asociadas a infección por sífilis en trabajadoras sexuales de la Ciudad de M éxico, 1993.

\begin{tabular}{|c|c|c|c|c|c|c|c|}
\hline Característica & $\mathrm{N} \cong$ & Prevalencia & $\mathrm{RM}^{*}$ & IC $95 \%$ & $\mathrm{RM} * *$ & IC $95 \%$ & Trend test \\
\hline \multicolumn{8}{|l|}{ Edad } \\
\hline 17 - 23 años & 230 & 4,8 & 1,00 & 1,00 & & & \multirow[t]{4}{*}{0,061} \\
\hline $24-30 “$ & 302 & 5,9 & 1,26 & $0,55-2,92$ & 1,23 & $0,54-2,78$ & \\
\hline 31 y mas & 262 & 9,9 & 1,97 & $0,77-5,20$ & 1,67 & $0,70-3,98$ & \\
\hline Total & 794 & 7,2 & & & & & \\
\hline \multicolumn{8}{|l|}{ Estado civil } \\
\hline Casada & 69 & 2,9 & 1,00 & 1,00 & & & \multirow[t]{4}{*}{0,309} \\
\hline Soltera & 465 & 6,7 & 2,39 & $0,54-14,81$ & 1,65 & $0,37-7,35$ & \\
\hline O tro & 254 & 7,8 & 2,59 & $0,56-16,50$ & 1,79 & $0,39-8,10$ & \\
\hline Total & 788 & 6,30 & & & & & \\
\hline \multicolumn{8}{|c|}{ Indice nivel socieconómico } \\
\hline NSE alto & 178 & 3,9 & 1,00 & 1,00 & & & \multirow[t]{4}{*}{0,016} \\
\hline NSE medio & 244 & 5,3 & 1,37 & $0,50-3,90$ & 1,09 & $0,41-2,87$ & \\
\hline N SE bajo & 351 & 9,1 & 2,45 & $1,01-6,23$ & 1,48 & $0,60-3,69$ & \\
\hline Total & 773 & 6,1 & & & & & \\
\hline \multicolumn{8}{|l|}{ Sitio de trabajo } \\
\hline Estética & 77 & 1,2 & 1,0 & 1,00 & & & \multirow[t]{4}{*}{0,001} \\
\hline Bar & 366 & 4,4 & 3,47 & $0,47-71,3$ & 3,56 & $0,41-31,1$ & \\
\hline Calle & 364 & 9,6 & 8,09 & $1,16-161,2$ & 5,54 & $0,68-44,7$ & \\
\hline Total & 807 & 5,1 & & & & & \\
\hline \multicolumn{8}{|c|}{ Antigüedad del trabajo } \\
\hline menos de 1 año & 223 & 4,0 & 1,0 & 1,00 & & & \multirow[t]{4}{*}{0,001} \\
\hline 15 años & 402 & 5,4 & 1,38 & $0,59-3,29$ & 1,16 & $0,51-2,62$ & \\
\hline 6 y más “ & 153 & 13,7 & 3,78 & $1,59-9,22$ & 2,31 & $0,91-5,86$ & \\
\hline Total & 778 & 7,7 & & & & & \\
\hline \multicolumn{8}{|c|}{ Clientes última semana } \\
\hline Ninguno & 350 & 4,6 & 1,00 & 1,00 & & & \multirow[t]{4}{*}{0,067} \\
\hline 1 - 10 clientes & 364 & 7,6 & 1,74 & $0,89-3,43$ & 1,09 & $0,44-2,70$ & \\
\hline 11 y más “ & 93 & 8,6 & 1,96 & $0,74-5,08$ & 1,64 & $0,50-5,36$ & \\
\hline Total & 807 & 6,9 & & & & & \\
\hline
\end{tabular}

* Razón de Momios Cruda

** Razón de M omios ajustada (edad, estado civil, N SE, sitio trabajo, antiguedad trabajo,clientes).

IC - Intervalo de confianza

Esto es atribuible a varios factores. Primero las prevalencias de ETS difieren según la región geográfica; segundo, las formas de organización del comercio sexual determinan el tipo de trabajadoras sexuales; y tercero existen diferentes técnicas que identifican diversos marcadores de una misma infección.

Sin embargo, los resultados obtenidos permiten ubicar la epidemiología de las ETS estudiadas en MTSC en el contexto mundial y nacional. En México, Valdespino y col. ${ }^{20}$ (1992), reportaron una prevalencia de infección por T. pallidum de 27,8 (VDRL/FTA-ABS) en 1.066 MTSC de 3 entidades federales del país. Gallegos ${ }^{8}(1993)$. reportó una prevalencia de $10,5 \%$ (VDRL) en 133 MTSC de Chihuahua. Uribe y col. ${ }^{19}(1993)$ reportaron una prevalencia de 6,6\% (RPR/FTA) en 2.918 MTSC en la ciudad de México. Finalmente, Juárez y col. ${ }^{11}$ (1992) reportaron una prevalencia de 2,3\% (RPR/ FTA) en 482 MTSC atendidas en un centro de CONASIDA.

Estos dos últimos resultados son similares a la prevalencia obtenida en este estudio, de 6,4\% (52/ $807)$, lo que sostiene que existe una prevalencia media-baja de este marcador serológico (T. pallidum) en MTSC de la ciudad de México, utilizando las mismas técnicas de laboratorio. Sin embargo, esta prevalencia es mayor a la encontrada en mujeres de población general. Por ejemplo, López ${ }^{12}$ (1987) reportó una prevalencia de 1,17\% (VDRL) en 1.362 donadores de sangre en Puebla; Zamilpa y col. ${ }^{21}$ (1994) reportaron una prevalencia de 2,5\% (VDRL/ FTA-ABS) en 389 mujeres que acudieron a consulta externa en Cuernavaca Mor. 
La importancia de resaltar la prevalencia de sífilis es que la presencia de esta infección facilita la infección de otras ETS como el VIH (Nakashima y col. ${ }^{14}$ 1992).

En general se trata de una población joven (media 29,2 años) con un promedio de 5 años de antiguedad en el trabajo y 7 clientes por semana; similares resultados se han obtenido en muestras de MTSC estudiadas en otros países. Por ejemplo en Australia la media de edad de 231 mujeres fue 28.3 años (Philpot y col. ${ }^{16}, 1991$ ); en Bélgica 154 mujeres tuvieron una media de edad, de antigüedad en el trabajo y de clientes en la última semana de 34,5 años, 7,1 años y 14 clientes, respectivamente (Mak \& Plum $\left.^{13}, 1991\right)$.

Los resultados obtenidos perfilan a identificar a un grupo de MTSC, con mayor probabilidad de presentar marcadores serológicos positivos para sífilis: Mujeres quienes trabajaban en puntos de calle (8 veces más), analfabetas (4 veces más) y quienes tenían una antigüedad en el trabajo mayor a 6 años (4 veces más).

En el análisis multivariado, los factores encontrados como predictores de riesgo de infección para sífilis, en este estudio, fueron NSE, sitio de trabajo, antigüedad en el trabajo y serología positiva para herpes tipo 2. Algunos autores han reportado resultados similares, como Celentano y col. ${ }^{6}$ (1994) quienes reportaron mayor riesgo de infección por VIH en MTSC mayores de 21 años, analfabetas, y antiguedad en el trabajo mayor a 2 años.

Finalmente, un os 53\% (432/807) de las MTSC manifestaron uso consistente del condón masculino con los últimos 3 clientes. Aunque los riesgos no mostraron significancia en esta muestra, se conoce que la utilización del condón masculino reduce hasta en el $50 \%$ la probabilidad de adquirir y transmitir alguna ETS.

Tomando en cuenta la población de estudio, pueden mencionarse las siguientes limitaciones del estudio: primero, por las connotaciones legales muchas mujeres trabajan de forma independiente y otras no se consideran trabajadoras sexuales, haciendo difícil estimar cuántas mujeres participan como trabajadoras sexuales; por consiguiente no existe un denominador confiable donde basar las estimaciones sobre prevalencia de ETS. Segundo, a pesar de tratarse de una entrevista confidencial donde se manejó la información mediante una clave que las identificara, hay resultados que no fueron consistentes (número de parejas regulares y clientes, uso de condón), lo que sugiere un posible sesgo de información. Tercero, en virtud de la baja prevalencia del evento $(6,4 \%=52$ mujeres $)$, la precisión de los estimadores calculados es baja, sin embargo muestran tendencias estadísticamente significativas.

\section{CONCLUSIONES}

En este grupo de mujeres existen condiciones inherentes a su trabajo (número de parejas y clientes, sexo sin protección) que hacen propicio el contagio y la propagación de diferentes ETS. También queda demostrado que existe una heterogenicidad de MTSC, diferenciado principalmente por el sitio donde se desempeñan.

Con la tendencia de incremento de casos heterosexuales de infecciones por VIH, es importante resaltar las medidas preventivas que las MTSC pueden usar. En nuestra sociedad el uso del condón está determinado por la decisión del compañero o cliente. Sin embargo hay estudios que demuestran que las MTSC están utilizando más el condón como medida protectora contra las ETS (Ameijden y col. ${ }^{1}, 1994$ ).

Debe entenderse que más que grupos en riesgo de adquirir y transmitir ETS, existen prácticas sexuales de riesgo en cualquier individuo que tiene relaciones sexuales, que aunadas a infecciones predisponentes como sífilis, facilitan la transmisibilidad de otras ETS.

Por lo tanto, las campañas de prevención y fomento de uso de condón, deben orientarse no sólo a las MTSC sino también a sus clientes y parejas, con la finalidad de que todos asuman su responsabilidad del sexo seguro. 


\section{REFERENCIAS}

1. AMEIJDEN, E.; VAN DEN HOEK, A.; VAN HAASTRECHT, H.; COUTINHO, R. Trends in sexual behaviour and the incidence of STD and HIV among drugusing prostitutes. Amsterdam 1986-92. AIDS, 8:213-21, 1994.

2. ANDRUS, J.; FLERNING, D.; HARGER, D.; CHIN, M.; BENNETT, D.; HORAN, J. et al. Partner notification: can it control epidemic syphilis. An. Int. Med., 12:539-43, 1990.

3. BRONFMAN, M.; GUISCAFRÉ, H.; CASTRO, V.; CASTRO, R.; GUTIÉRREZ, G. Medición de la desigualdad: una estrategia metodológica, análisis de las características socioeconómicas de la muestra. Arch. Invest. Med., 19:35-40, 1988.

4. BROWN, S.; ZACARIAS, F.; ARAL, S. STD control in less developed countries: the time is now. Int. J. Epidem., 14:505-9,1985.

5. CATANIA, J.; COATES, T.; STALL, R.; TURNER, H.; PETERSON, J.; HEARST, N. et al. Prevalence of AIDS related risk factors and condom use in the United States. Science, 258:1101-6, 1992.

6. CELENTANO, D.; AKARASEWI, P.; SUSSMAN, L.; SUPRASERT, S.; MATANASARAWOOT, A WRIGHT, N. et al. HIV-l infection among lower class commercial sex workers in Chiang Mai, Thailand. AIDS, 8:533-7, 1994.

7. ESTÉBANEZ, P.; FITCH, K.; NÁJERA, R. El VIH y las trabajadoras sexuales. Bol. Of. Sanit. Panam., 15:415-35, 1993.

8. GALLEGOS, F. Experiencia en la detección de enfermedades de transmisión sexual en un grupo de mujeres prostitutas. In: Congreso Nacional de Investigación en Salud Pública, $4^{\circ}$. Cuernavaca Mor. 1993. Cuaderno de trabajo p. 63.

9. GÓMEZ, R.; ARANGO, M.; VELÁZQUEZ, G.; OROZCO, $B$. Factores de riesgo de infección en usuarios de un programa de control del VIH, Antioquía, Colombia. Bol. Of. Sanit. Panam., 108:181-91, 990.

10. JUÁREZ, L.; HERNÁNDEZ, P.; CONDE, C.; ALLEN, B. Procedure manual: laboratory diagnosis of STD's. México, Centro de Investigaciones sobre Enfermedades Infecciosas, Instituto Nacional de Salud Pública. Cuernavaca Morelos, 1992.

11. JUÁREZ, L.; URIBE, F; HERNÁNDEZ, P.; URIBE, P.; HERNÁNDEZ, G.; CONDE, C. et al. Prevalence and determinants of HIV and other STD's in a population of female commercial sex workers in Mexico city. In: International Conference on AIDS, $8^{\circ}$ STD World Congress, $3^{\circ}$ Amsterdam, 1992. Abstract PoC 4131.
12. LÓPEZ, M. Prevalencia de anticuerpos contra el VIH y otros marcadores serológicos de enfermedades infecciosas en donadores de sangre en la ciudad de Puebla, México. Rev. Invest. Clin., 39:219-22,1987.

13. MAK, R. \& PLUM, J. Do prostitutes need more health education regarding STD's and the HIV infection? Experience in a Belgian city. Soc. Sci. Med., 33:963-6, 1991.

14. NAKASHIMA, K.; KASHIWAGI, S.; HAYASHI, J.; NOGUCHI, A.; HIRATA, M.; KAGIYAMA, W. et al. Sexual transmission of hepatitis $\mathrm{C}$ virus among female prostitutes and patients with sexually transmitted diseases in Fukuoka, Kyushu, Japan. Am. J. Epidem., 136:1132-7, 1992.

15. ORGANIZACIÓN PANAMERICANA DE LA SALUD. Informe anual de vigilancia del SIDA, VIH y ETS para la región de Las Américas, 1992. Washington, D.C. 1994.

16. PHILPOT, C.; HARCOURT, C.; EDWARDS, J. A survey of female prostitutes at risk of HIV infection and other STD. Genitourin. Med., 67:384-8, 1991.

17. PIOT, P. \& TEZZO, R. The epidemiology of HIV and other sexually transmitted infections in the developing world. Scan. J. Infect. Dis. Suppl., 69:89-97, 1990.

18. RAWSTRON, S.; JENKINS, S.; BLANCHARD, S.; PINGWU, L.; BROMBERG, K. Maternal and congenital syphilis in Brooklyn NY. Epidemiology, transmission, and diagnosis. AJDC, 147:727-31, 1993.

19. URIBE, F.; HERNÁNDEZ, M.; JUÁREZ, L.; HERNÁNDEZ, P.; CONDE, C.; URIBE, P.; et al. Prevalencia y determinantes de algunas ETS en personas con comportamiento sexual de alto riesgo en la ciudad de México. In: Congreso Nacional de Investigación en Salud Pública, $4^{\circ}$. Cuernavaca Mor., 1993. Cuaderno de trabajo p. 76.

20. VALDESPINO, J.L.; GARCÍA, M.; LOO, E.; SALCEDO, R.; MAGIS, C.; CRUZ, C.; et al. HIV-1 and STD centinel surveillance among homosexual men and female prostitutes in Mexico. In: International Conference on AIDS $/ 8^{\circ} / \mathrm{STD}$ World Congress, $3^{\circ}$ Amsterdam, 1992 Abstract PoC 4052.

21. ZAMILPA, L.; RIVERA, L.; HEMÁNDEZ, P.; CONDE, C. Prevalencia de factores asociados a sífilis y herpes genital en un grupo de población general femenina. Enferm. Infec. Microbiol.,14:267,1994.

22. ZENKER, P. \& ROLFS, R. Treatment of syphilis. Rev. Infect. Dis. Suppl., 6:590-606, 1990. 